\title{
An analysis of the characteristics of sports activities and injury experiences of leisure sports participants
}

\author{
Jong-Soon Kim', Hye-Sang Park', Sae-Sook Oh, ${ }^{1, *}$ \\ 'Department of Leisure Sport, Kyonggi University, Suwon, Korea \\ ${ }^{2}$ Department of Physiology, College of Medicine, Kyung Hee University, Seoul, Korea
}

The purpose of this study is to analyze exercise injury experiences of sports participants and the characteristics of sporting activities depending on whether they have injured or not. The subjects of this study included the 107 people who have experienced exercise injuries and the 103 people who have not have experienced exercise injuries with aged between the 20 s and 40 s. The questionnaire with household interviews was used for this research. The results of this study that the female participants who have experienced an injury while exercise was outnumbered by their male counterparts and it is found that sports participants in their 40 s represented the highest ratio and that the two most popular sports the participants who have experienced injury did were body-building and golf. It has been surveyed that the number of hospital care due to exercise injury per year is 2.01 times on average and the amount of money spent on the medical care is 34,252 Korean won on average. It is identified that the level of sports activities that keeping health and perception by the impact of sports activities depending on the participants' exercise injured. It is worthy of notice that those who have experienced exercise injuries tended to show a high level of confidence in which they could benefit from sports activity. In addition, it is interesting to note that bodybuilding and golf were the two sports with a great number of exercise injuries.

Keywords: Leisure sports, Activities, Injury, Experience

\section{INTRODUCTION}

Participation sports out of leisure activities are voluntary and ordinary activities to develop one's health and fitness. Participating sports activities, according to 2016 National Report on Leisure Activities, was the third most popular leisure type which was only preceded by rest (watching TV etc.) and hobby \& amusement (collection, crafts, etc.) (Ministry of Culture, Sports and Tourism, 2016). There has so far been little interest in scientific research into participation sports and such programs as injury experiences and rehabilitation services, given the rising popularity of participation sports in South Korea. So, it is an important moment when exercise injury experiences should be surveyed and analyzed by way of a national survey on citizen's sports participation. The causes of exercise injuries are varied: a lack of skills and of warm-up, an excessive amount of exercise and accumulated fa- tigue, high tension and decreasing attention, and foul plays and violent activities for instance (Ham and Jee, 2016; Kim and Ju, 2013; Tracy, 2003). There are a number of studies on those cases, most of which particularly focused on injury prevention, the causes of injuries and treating injuries in a particular sport for the sake of protecting elite athletes. However, a couple of researches into exercise injuries of amateur sports participants were conducted with the subjects of assessing the causes of injuries by different parts of bodies (Batt, 1992; Lee et al., 2012). There was a study on the relationship between the level and duration of training, and injuries in a particular sport (Gabbett, 2004; Ham and Jee, 2016). In addition, there was an academic monograph on the causes of injuries and their patterns by divers' education grade levels (Hyun et al., 2015). A research on adolescent physical activity-related injuries in such different settings as a sports club, school sports, and other leisure time activities is also noteworthy
${ }^{\star}$ Corresponding author: Sae-Sook Oh (D) https://orcid.org/0000-0001-8880-406X Department of Leisure Sport, Kyonggi University, 154-42 Gwanggyosan-ro, Yeongtong-gu, Suwon 16227, Korea

Tel: +82-31-249-9980, Fax: +82-31-249-9075, E-mail: sxo139@gmail.com Received: April 1, 2018 / Accepted: May 9, 2018
This is an Open Access article distributed under the terms of the Creative Commons Attribution Non-Commercial License (http://creativecommons.org/licenses/by-nc/4.0/) which permits unrestricted non-commercial use, distribution, and reproduction in any medium, provided the original work is properly cited. 
(Räisänen et al., 2016). Yet, precedent studies aforementioned mainly related to the causes and symptoms of exercise injuries. In light of this, it seems important to analyze sports participants' injury experiences in a wider perspective utilizing national survey. This study aims at analyzing exercise injury experiences of sports participants aged between the 20s and 40s and the characteristics of sporting activities based on their injury experiences. It would seek to provide basic information to vitalize exercise rehabilitation and prescription, and counseling service by assessing the characteristics of their sports activities depending on their exercise injury experiences and the conditions of injury experiences.

\section{MATERIALS AND METHODS}

\section{Participants}

The original source for this study was the 2016 Survey on Citizen's Sports Participation in South Korea. The objects of this study included 189 persons (2.1\%) responding that they have experienced injuries and another 189 persons out of $6,065(67.3 \%)$ who did not experience any injuries were randomly sampled. The respondents who answered the 2016 Survey on Citizen's Sports Participation in South Korea were 9,012 with 2,758 missing value. As indicated in Fig. 1, the 107 people who have experienced exercise injuries and the 103 people who did not experience exercise injuries were used for this study. They were all aged between the 20 s and the 40s. The reason for excluding participants over 50s lay in the fact that only a small number of them have experienced exercise injuries with 19 (50s), 7 (60s), and 4 (70s) (Fig. 1). The social demographic characteristics of this study were as seen in Table 1: the number of male participants was 125 (59.5\%), and that of female counterparts was 85 (40.5\%), and the numbers of participants in their the 40s and the 30s are respectively the highest, 86 and the lowest, 59 which account for $41 \%$ and $28.1 \%$ (Table 1).

\section{Experimental procedures}

This survey is annually conducted for the purposes of thoroughly understanding nation's needs for sports activities, of minimiz-

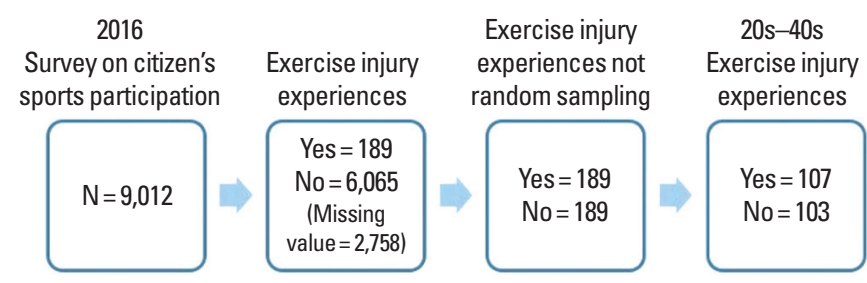

Fig. 1. The process of the sampling. ing constraints on participating sports activities, and of maximizing enhancement of participation sports (Ministry of Culture, Sports and Tourism, 2016). It should be noted that this study attempted to overcome such problems as regional differences, representativeness of those questioned, and suitability of sampling methods by utilizing the original source, the data of nationwide unit through probability sampling. A structured questionnaire with household interviews was used for this research. The way of interviews is to employ Paper and Pencil by visiting relevant household and selecting an eligible one person per household. The procedure of data processing and verification was, in turn, progressed with investigators' self-verification, investigating managers' verification, logical fallacy verification, and comparative data verification. Only valid samples excluding unreliable questionnaires were used for data input in accordance with a coding manual. For the final analysis, relevant questions on exercise injury experiences of sports participants aged between the 20s and the 40s, and on the characteristics of their sports activities were selected.

\section{Measurements tools}

The conditions of exercise injury experiences comprised the assessment of the participants who previously have experienced exercise injuries by their sex, age, favorite sports programs, the number of their visits to the hospital on account of exercise injuries during the last year, their medical expenses per visit and their changes in sports activities after experiencing injuries. The first characteristics of their sports activities identified through a questionnaire comprise their regular sports activities for keeping their physical fitness and health, enough rest and sleep, regular meal and replenishing nourishment, and whether they smoke and drinks. To collect the data on these, relevant questions that how you do regularly sports activities for keeping your physical fitness

Table 1. Socio-demographic characteristics of participants

\begin{tabular}{lr}
\hline Characteristic & No. $(\%)$ \\
\hline Exercise injury experiences & \\
Yes & $107(51.0)$ \\
No & $103(49.0)$ \\
Sex & \\
Male & $125(59.5)$ \\
Female & $85(40.5)$ \\
Age & \\
20 s & $65(31.0)$ \\
$30 s$ & $59(28.1)$ \\
$40 s$ & $86(41.0)$ \\
Total & $210(100)$ \\
\hline
\end{tabular}


and health, for instance, were used through Likert five-point scale: one point for never fulfilling at all and five points for fulfilling very well. The second characteristics of their sports activities identified through a questionnaire are about the duration and intensity of sports programs in which they mainly participate. An open-ended question was used to obtain data on the duration of sports programs in which they mainly participate: 'how many months do you participate in a sport over the last year?' As to the intensity of sports programs, a question also used by way of threepoint scale: one point for low and three points for high: "how was the intensity of sports activities in which you mainly participate?' The third characteristics of their sports activities identified through a questionnaire are linked with the effect of maintaining their physical and psychological health by doing regular sports activities. A relevant question - "how much impact of sports activities do you think on keeping your physical and psychological health?' - is used by way of Likert five-point scale with one point for 'never effective at all' and 5 points for 'very effective.'

\section{Statistical analyses}

The frequency analysis was employed to grasp the social demographic characteristics and the conditions of exercise injury experiences of the participants. The $t$-test was also employed to analyze the level of health keeping fulfillment, of sports activities, and of the impact through sports activities. IBM SPSS Statistics ver. 20.0 (IBM Co., Armonk, NY, USA) was used for all the analyses and the statistical reliability was estimated at $\alpha=0.05$.

\section{RESULTS}

\section{The conditions of exercise injury experiences}

To investigate the conditions of exercise injury experiences this study assessed the characteristics of 107 persons aged between the 20 s and the 40s who previously have experienced exercise injuries by their gender, age, favorite sports programs, the number of their visits to hospital on account of exercise injuries during the last year, and their medical expenses per visit as shown in Table 2. As to exercise injury experiences the number of male participants was higher than that of their female counterparts. The participants in the 40s who have experienced exercise injuries accounted for the largest proportion of $45.8 \%$ of 49 respondents, while the participants in their 20s accounted for the smallest proportion of $25.2 \%$ of 20 respondents. Also, the major sports programs of the participants with injury experiences were reported as follows: the 15 participants answered bodybuilding and golf respectively, which
Table 2. The conditions of exercise injury experiences

\begin{tabular}{lc}
\hline Characteristic & Value \\
\hline Sex & \\
Male & $77(72)$ \\
Female & $30(28)$ \\
Age & \\
20 s & $20(25.2)$ \\
30 s & $31(29)$ \\
40 s & $49(45.8)$ \\
Sports & \\
Body building & $15(14)$ \\
Golf & $15(14)$ \\
Walking & $10(9.3)$ \\
Cycling & $8(7.5)$ \\
Football & $8(7.5)$ \\
Yoga & $7(6.5)$ \\
Badminton & $6(5.6)$ \\
Swimming & $5(4.7)$ \\
Basketball & $4(3.7)$ \\
Etc. & $28(27.1)$ \\
No. of the participants' hospital visits due to injuries & $2.01 \pm 1.356(1-8)$ \\
Medical expenses per visit (KRW) & $34,252 \pm 58,446(4,000-50,000)$ \\
Achange in their participation in sports after injuries & $2.90 \pm 0.454(1-4)$ \\
Total & $107(100)$ \\
\hline
\end{tabular}

Values are presented as number (\%) or mean standard \pm deviation (range). KRW, Korean won (the currency of South Korea).

was followed by walking, bicycle, and football. The number of their visits to the hospital on account of exercise injuries during the last year was 2.01 times on average and medical expenses per visit were 34,252 Korean won (KRW). Changes in their participation in sports activities after experiencing injuries were analyzed at 2.9 on average, which was tantamount to 3.0 with a meaning of 'no change.'

\section{The characteristics of sports activities depending on participants' injury experiences}

It is analyzed that there were differences in the characteristics of sports activities including the degree of sports activities, of maintaining health, and of perception on the impact of sports activities depending on participants' exercise injury experiences as we shall see Table 3. There was no statistical difference between exercise injury experiences and the number of the month the participants have done sports activities. There was a statistically significant difference between exercise injury experiences and the intensity of the participation in sports $(P<0.001)$. Those who have experienced exercise injuries indicated higher intensity in their partici- 
Table 3. An analysis on the differences of the characteristics of sportss activities depending on participants' injury experiences

\begin{tabular}{|c|c|c|c|}
\hline Variable & Mean \pm SD & $t$ & $P$-value \\
\hline \multicolumn{4}{|l|}{ Degree of sports activities } \\
\hline \multicolumn{2}{|l|}{ No. of month participating in sports activities } & 1.082 & 0.281 \\
\hline Yes & $8.60 \pm 3.515$ & & \\
\hline No & $8.07 \pm 3.587$ & & \\
\hline \multicolumn{2}{|c|}{ Degree of intensity in their participation in sports } & 4.287 & $0.000^{* * *}$ \\
\hline Yes & $2.23 \pm 0.592$ & & \\
\hline No & $1.89 \pm 0.559$ & & \\
\hline \multicolumn{4}{|l|}{ Degree of maintaining health } \\
\hline Regular participation in sports activities & & 2.575 & $0.011^{*}$ \\
\hline Yes & $3.75 \pm 0.778$ & & \\
\hline No & $3.46 \pm 0.861$ & & \\
\hline Enough rese and sleep & & -2.745 & $0.007^{* *}$ \\
\hline Yes & $3.38 \pm 0.928$ & & \\
\hline No & $3.71 \pm 0.788$ & & \\
\hline \multicolumn{2}{|l|}{ Regular meal and replenishing nourishment } & -2.142 & $0.033^{*}$ \\
\hline Yes & $3.54 \pm 0.974$ & & \\
\hline No & $3.81 \pm 0.805$ & & \\
\hline Temperance and no smoking & & -3.356 & $0.001^{* * *}$ \\
\hline Yes & $3.17 \pm 1.059$ & & \\
\hline No & $3.68 \pm 1.148$ & & \\
\hline \multicolumn{4}{|l|}{ Degree of the perception with sports activities } \\
\hline Keeping physical health & & 2.117 & $0.035^{*}$ \\
\hline Yes & $4.22 \pm 0.619$ & & \\
\hline No & $4.05 \pm 0.584$ & & \\
\hline Keeping psychological health & & 2.790 & $0.006^{* *}$ \\
\hline Yes & $4.28 \pm 0.626$ & & \\
\hline No & $4.03 \pm 0.678$ & & \\
\hline
\end{tabular}

$\mathrm{SD}$, standard deviation.

${ }^{*} P<0.05 .{ }^{* *} P<0.01 .{ }^{* *} P<0.001$.

pation in sports than those who did not experience any injuries.

The difference between exercise injury experiences and the degree of sports activities is as follows. It is found that there was a statistically significant difference between them, subject to participants' injury experiences $(P<0.05)$. There was a tendency that those who have experienced injuries more regularly did sports activities than those who did not experience any injuries. There was also a statistically significant difference between exercise injury experiences and the degree of enough rest and sleep $(P<0.01)$. Those who did not experience injuries tended to take enough rest and sleep on a regular basis compared to those who have experienced injuries. There was a statistically significant difference between the degree of having a regular meal and of replenishing nourishment, and participants' injury experiences $(P<0.05)$. Those who did not experience injuries tended to have regularly their meals and nourishment compared to those who have experienced injuries. There was a statistically significant difference between the habit of smoking and drinking and participants' injury experiences $(P<0.001)$. Those who have experienced injuries were more exposed to smoking and drinking compared to those who did not experience any injuries.

The difference between exercise injury experiences and the degree of the impact through sports activities is as follows. There was a statistically significant disparity between the degree of perception on the effect of keeping physical health through sports activities and participants' injury experiences $(P<0.05)$. It is identified that those who have experienced exercise injuries recognized, more importantly, the impact of keeping physical health through sports activities than those who did not experience exercise injuries. There was a statistically significant gap between the degree of perception on the effect of keeping psychological health through sports activities and participants' injury experiences $(P<0.01)$. It is identified that those who have experienced exercise injuries recognized, more importantly, the impact of keeping physical health through sports activities than those who did not experience exercise injuries.

\section{DISCUSSION}

The results of this study can be summarized as follows. First, as to exercise injury experiences, the number of male participants was higher than that of their female counterparts and the participants in the 40s accounted for the largest proportion. Sports programs in which the participants with injury experiences mainly participated were bodybuilding and golf. The number of visits to the hospital due to exercise injuries over the last year was about 2 times on average and medical expenses spent per visit was over 30,000 KRW. Also, many of those who have experienced injuries replied that their participation in sports activities after suffering injuries was not changed. Second, those who have experienced exercise injuries showed higher intensity in their participation in sports than those who did not experience any injuries. Also, the former more regularly did sports activities than the latter. Besides, the latter took sufficient rest and sleep and more regularly had a meal and supplemented nourishment than the former. It is identified that the latter showed a lower level of smoking and of drinking compared to the former. Finally, those who have experienced exercise injuries recognized, more importantly, the impact of keeping physical and psychological health through sports activities than those who did not experience exercise injuries. 
The relationship between the characteristics of sports activities and exercise injury experiences based on the results of this study could be suggested as follows. First, it needs to bring attention to the characteristics of particular sports programs to reduce exercise injuries in the South Korean sports-for-all domain in that a large number of bodybuilding and golf participants have experienced injuries. When it comes to weightlifting, the extent of participants' injuries basically depends on their age and their purpose of exercise (Jones et al., 2010). In addition, fitness activity-related injury prevention strategies, according to Gray and Finch (2015), should be targeted at different subgroups based on the type of fitness activity being undertaken. In the meantime, as to golf, the amount of gameplay and the last time clubs were changed were significantly associated with the risk of golf injury (McHardy et al., 2007). It should be also noted that the popularity of bodybuilding is high and the number of bodybuilding participants has been rising: in 2015, bodybuilding with a 2.6\% increase compared with the previous year was the third popular sports in South Korea, which was preceded by walking and mountaineering (Ministry of Culture, Sports and Tourism, 2016). It would be unusual that a large number of golf participants have experienced injuries although golf is not a strenuous sport with no physical contact. However, a research undertaken by Batt (1992) is worthy of our consideration. He has argued that exercise injury ratio of a particular sport could go up even if the sport is not a physically hard and intense sport. Indeed the number of golf participants. Under this context, even greater interest in the treatment and rehabilitation programs for the two sports seems to be required. Second, those who have experienced exercise injuries showed a higher degree of exercise intensity and did regularly sports activities than those who did not experience any injuries. The former recognized, more importantly, the impact of keeping physical and psychological health through sports activities than the latter. In light of this, it could be presumed that the former has a high degree of confidence in which they could benefit from sports activity. In other words, their positive attitudes reflect beliefs that physical activity offers some rewards (Nelson et al., 2010). It could also be analyzed that their positive attitudes toward sports activities could be considered as perceived benefits (Rundle-Thiele et al., 2016). Apart from health benefits, physical activity could be associated with health particular injury risk is greatest when individuals perform at the highest levels of relative intensity (Khan et al., 2012).

There were constraints of this study in that it referred to only relevant questions having been used for the 2016 Survey on Citi- zen's Sports Participation in South Korea in assessing exercise injury experiences. Henceforth further researches with more segmented questions, by age groups, sports programs, and the level of regular sports activities, for instance, would be required to help to prevent and reduce exercise injuries in the South Korean participation sports domain. In order to increase participation in sports and widen the health benefits of the public, it is critical to eliminate the barriers to sporting activities such risk factors as exercise injuries and in this context, sports medicine could play an important role.

\section{CONFLICT OF INTEREST}

No potential conflict of interest relevant to this article was reported.

\section{REFERENCES}

Batt ME. A survey of golf injuries in amateur golfers. Br J Sports Med 1992; 26:63-65.

Gabbett TJ. Influence of training and match intensity on injuries in rugby league. J Sports Sci 2004;22:409-417.

Gray SE, Finch CF. Epidemiology of hospital-treated injuries sustained by fitness participants. Res Q Exerc Sport 2015;86:81-87.

Ham DW, Jee YS. Yachters in Korea suffer considerable injuries. J Exerc Rehabil 2016;12:226-231.

Hyun GS, Jee YS, Park JM, Cho NH, Cha JY. Injury survey in scuba divers of British Sub-Aqua Club: a retrospective study. J Exerc Rehabil 2015; 11:331-336.

Jones C, Hammig B, Henry J. Weightlifting injuries in the USA: data from 2000 to 2008. Inj Prev 2010;16:A84.

Khan KM, Thompson AM, Blair SN, Sallis JF, Powell KE, Bull FC, Bauman AE. Sport and exercise as contributors to the health of nations. Lancet 2012;380:59-64.

Kim HY, Ju SB. The index analysis of hip displacement of middle school fencers and students. J Korean Soc Rhythmic Exer 2013;6:23-31.

Lee JY, Chang MJ, Choi HY, Lee SE. Actual situation of the participants involved physical living exercise sports injury research in accordance. J Sport Leis Stud 2012;49:701-728.

McHardy A, Pollard H, Luo K. One-year follow-up study on golf injuries in Australian amateur golfers. Am J Sports Med 2007;35:1354-1360.

Ministry of Culture, Sports and Tourism. Survey on citizen's sports participation. Sejong (Korea): Ministry of Culture, Sports and Tourism; 2016.

Nelson TD, Benson ER, Jensen CD. Negative attitudes toward physical 
activity: measurement and role in predicting physical activity levels among preadolescents. J Pediatr Psychol 2010;35:89-98.

Räisänen AM, Parkkari J, Karhola L, Rimpelä A. Adolescent physical activity-related injuries in sports club, school sports and other leisure time physical activities. Cogent Med 2016;3:1260786.
Rundle-Thiele S, Kubacki K, Gruneklee N. Perceived benefits and barriers of physical activity: a social marketing formative study. Health Mark Q 2016;33:181-194.

Tracy J. The emotional response to the injury and rehabilitation process. J Appl Sport Psychol 2003;15:279-293. 